\title{
Dietary fibre intake and its associations with depressive symptoms in a prospective adolescent cohort
}

\author{
Olivia G. Swann ${ }^{1}$, Monique Breslin ${ }^{1}$, Michelle Kilpatrick ${ }^{1}$, Therese A. O'Sullivan ${ }^{2}$, Trevor A. Mori ${ }^{3}$, \\ Lawrence J. Beilin ${ }^{3}$, Ashleigh $\operatorname{Lin}^{4}$ and Wendy H. Oddy ${ }^{1 *}$ \\ ${ }^{1}$ Menzies Institute for Medical Research, University of Tasmania, Hobart, TAS 7000, Australia \\ ${ }^{2}$ School of Medical and Health Science, Edith Cowan University, Joondalup, WA 6027, Australia \\ ${ }^{3}$ Medical School, The University of Western Australia, Perth, WA 6000, Australia \\ ${ }^{4}$ Telethon Kids Institute, The University of Western Australia, Nedlands, WA 6009, Australia
}

(Submitted 4 May 2020 Final revision received 24 August 2020 Accepted 24 August 2020)

\section{Abstract}

Depression is a major cause of disability in adolescents. Higher dietary fibre intake has been associated with lower depressive symptoms in adults, but there is a lack of research in adolescents. We examined the association between dietary fibre intake (Commonwealth Scientific and Industrial Research Organisation (CSIRO) FFQ) and depressive symptoms (Beck Depression Inventory for Youth) in adolescents with pro spective data from the Raine Study Gen 214 and 17 year follow ups ( $n 1260$ and 653). Odds of moderate/extreme (dinically relevant) depres sive symptoms by quartile of fibre intake were calculated using mixed effects logistic regression for all participants, in a paired sample without moderate/extreme depressive symptoms at 14 years and in a sub sample of participants with available inflammatory data at the ages of 14 and 17 years ( $n 718$ and 547). Odds of moderate/extreme depressive symptoms were lower in the fourth (highest) quartile of overall fibre intake (OR $0.273,95 \% \mathrm{CI} 0.09,0 \cdot 81$ ) compared with the first (lowest) quartile, adjusting for sex, age, energy intake, adiposity, and family and lifestyle factors. However, further adjustment for dietary patterns attenuated the results. Associations of depressive symptoms with cereal or fruit and vegetable fibre intake were not significant in the final model. Adjustment for inflammation had no effect on OR. The association between a higher dietary fibre intake and lower odds of clinically relevant depressive symptoms may be more reflective of a high fibre diet with all its accom panying nutrients than of an independent effect of fibre.

Key words: Dietary fibre: Inflammation: Adolescence: Depressive symptoms: Raine Study

Depression is a major cause of disability, with depressive disor ders thought to affect over 260 million people worldwide ${ }^{(1)}$. This is particularly evident in adolescents and young adults, with uni polar depression being the most common contributor to disabil ity between the ages of 10 and 24 years ${ }^{(2)}$. Maintaining a healthy diet, including a diet high in fruit and vegetable intake, may reduce risk of depression and is associated with a decrease in depressive symptoms ${ }^{(3-9)}$. Meta analysis of randomised con trolled trials consisting of whole of diet interventions has shown significantly reduced depressive symptoms with dietary inter vention $^{(10)}$. Additionally, intervention studies focusing on spe cific food groups such as fruits, vegetables and wholegrains show potential to reduce severity of depressive symptoms ${ }^{(11,12)}$. These plant foods are often high in dietary fibre.

Observational studies have shown that adults with low fibre diets have greater severity of depressive symptoms ${ }^{(13-19)}$. This association is seen with total fibre along with fruit, vegetable and cereal fibre. However, only one study to date has focused on adolescence ${ }^{(20)}$ despite evidence that up to $50 \%$ of adult mental health disorders first emerge during this period ${ }^{(21,22)}$.

Understanding and treating mental health concerns in adoles cence is particularly important due to the long term ongoing effects associated with depression ${ }^{(23)}$. Having juvenile or adoles cent depression has been associated with higher odds of adult anxiety, depression, mania and schizophreniform disorder ${ }^{(22)}$. Even subthreshold depressive symptoms in adolescence have been associated with higher rates of major depression and sui cidal tendencies in adulthood ${ }^{(24)}$. Proximal correlates of adoles cent depression include impaired school performance and attendance, and a higher likelihood of engaging in unhealthy behaviours such as smoking and binge eating ${ }^{(25,26)}$. These factors associated with depression may have detrimental consequences

Abbreviations: BDI Y, Beck Depression Inventory for Youth; CSIRO, Commonwealth Scientific and Industrial Research Organisation; hs CRP, high sensitivity $C$ reactive protein; VIF, variance inflation factor; $\mathrm{WHtR}$, wa ist:height ratio.

* Corresponding author: Professor Wendy H. Oddy, email Wendy.Oddy@utas.edu.au 
for future employment and health. It has been suggested that early treatment of depression, including during adolescence, may improve outcomes ${ }^{(27,28)}$.

The mechanisms by which dietary fibre may impact depres sion are currently unclear, but adiposity may be involved. Waist circumference and abdominal adiposity have been linked to depressive symptoms ${ }^{(29)}$, and increased risk of mental disorders in adulthood has been associated with being overweight in childhood $^{(30)}$. These relationships between adiposity and depression are likely to be bidirectional. Depression has been associated with cravings for foods high in sugar and saturated fat which may contribute to increases in adiposity ${ }^{(31,32)}$. On the other hand, the release of pro inflammatory cytokines with adiposity may play a role in the link between overweight and obesity and depression ${ }^{(33-35)}$. Inflammation is also a potential mediator of the association between dietary fibre and depressive symptoms. Studies have linked a high dietary fibre intake with decreased chronic inflammation ${ }^{(36-38)}$ and the presence of elevated inflammation with depression ${ }^{(39-43)}$. An alternative explanation for a relationship between dietary fibre and depres sion may be the effects of other nutrients obtained from foods high in dietary fibre.

The direction of the relationship between dietary fibre intake and depression is unclear. Higher intake of fat and sugar dense foods which are likely to be low in dietary fibre can be seen in those with depression ${ }^{(31,32)}$. However, there is evidence that people with previous depression may have healthier diets than those without previous depression ${ }^{(44)}$. Our hypothesis is that the population averaged effect is in the direction from diet to depression.

Thus far, the association of dietary fibre intake with depres sion is an understudied area, particularly in adolescents. In our study, we aimed to seek evidence for the beneficial effect of dietary fibre intake on depressive symptoms in adolescents at 14 and 17 years of age and to investigate the impact of adiposity and inflammation on that relationship. The confounding effect of other nutrients present in high fibre foods was considered.

\section{Methods}

Population

The Raine Study recruited 2900 pregnant women (termed Generation 1) from King Edward Memorial Hospital in Perth, Western Australia. The resulting 2868 babies (Generation 2) have been regularly followed up from birth. Detailed cohort information can be found elsewhere ${ }^{(45,46)}$. There were 1864 ado lescents participating in the 14 year follow up and 1726 in the 17 year follow up. After excluding those with incomplete data on all covariates except inflammation, 1260 adolescents in the 14 year follow up were included in principle analyses and 653 from the 17 year follow up (Fig. 1). Of these adolescents, 531 had complete data at age 14 and 17 years after excluding those with moderate/extreme depressive symptoms at the 14 year fol low up and were used in secondary analysis. A subset of partic ipants restricted to those with data on the inflammatory marker high sensitivity $\mathrm{C}$ reactive protein (hs CRP) included 718 adoles cents at the 14 year follow up and 547 at the 17 year follow up.

\section{Ethics}

The present study was conducted according to the guidelines in the Declaration of Helsinki. Ethics approval for the Raine Study was granted by the committees of King Edward Memorial Hospital for Women and Princess Margaret Hospital for Children, Perth, Western Australia. The adoles cent and their primary caregiver provided informed written consent.

\section{Diet measurement}

Dietary fibre intake and other dietary data were collected using the 212 item Commonwealth Scientific and Industrial Research Organisation (CSIRO) FFQ. The CSIRO FFQ is a semi quantitative self report FFQ measuring usual diet over the past year. Studies have validated the CSIRO FFQ for use in the Australian population and in Raine Study adolescents specifically ${ }^{(47-49)}$. Comparison of the CSIRO FFQ to a $3 \mathrm{~d}$ food record in the Raine Study at the 14 year follow up found a moderate positive correlation for dietary fibre $(r 0.43$ in males and $r 0.29$ in females, $P<0.001$ for both $)^{(47)}$. Participants were asked to estimate frequency and quantity of intake of foods based on provided standard serving size examples (e.g. one serve of pasta is one cup of cooked pasta). The serving size and frequency of consumption of each food item were used by the CSIRO to calculate estimated daily nutrient intakes. Dietary fibre is defined as fibre obtained from whole foods (overall fibre), from cereal and grains (cereal fibre) and from fruits or vegetables (fruit and vegetable fibre). Intakes of all fibre types were divided into quartiles for analysis as decided a priori. Available nutrients associated with depressive symptoms in the literature and in this sample include potas sium, $\mathrm{Mg}, \mathrm{Fe}, \mathrm{Cu}, \mathrm{Zn}$, thiamine, riboflavin, vitamin $\mathrm{B}_{6}$ and folate ${ }^{(16,20,50-52)}$. Dietary patterns termed 'Healthy' and 'Western' have been determined previously in the Raine Study as described elsewhere ${ }^{(53)}$. In brief, the 212 foods mea sured in the questionnaire were merged into thirty eight food groups which were used in factor analysis to identify data driven dietary patterns. The Healthy pattern includes con sumption of high fibre foods such as wholegrain cereals, fresh fruit, legumes and vegetables, along with steamed, grilled or canned fish. The Western pattern includes takeaway foods, red and processed meats, full fat dairy products and other refined foods ${ }^{(53)}$ (see reference for full list of components).

\section{Inflammation measurement}

Serum levels of hs CRP were used as a marker of inflammation. Participants with hs CRP concentrations over $10 \mathrm{mg} / 1$ were excluded as these concentrations represent acute inflammation and the present study aimed to examine the impact of chronic inflammation $(<10 \mathrm{mg} / \mathrm{l})$. Twelve hour fasting blood samples were taken by a trained home visiting phlebotomist early in the morning and analysed at the PathWest Laboratory at the Royal Perth Hospital. hs CRP was measured on an Architect c16000 analyser with an intra assay CV of $15.96 \%$ and a lower detection threshold of $0.15 \mathrm{mg} / \mathrm{l}$. Values of hs CRP were $\log$ transformed due to skewness. 


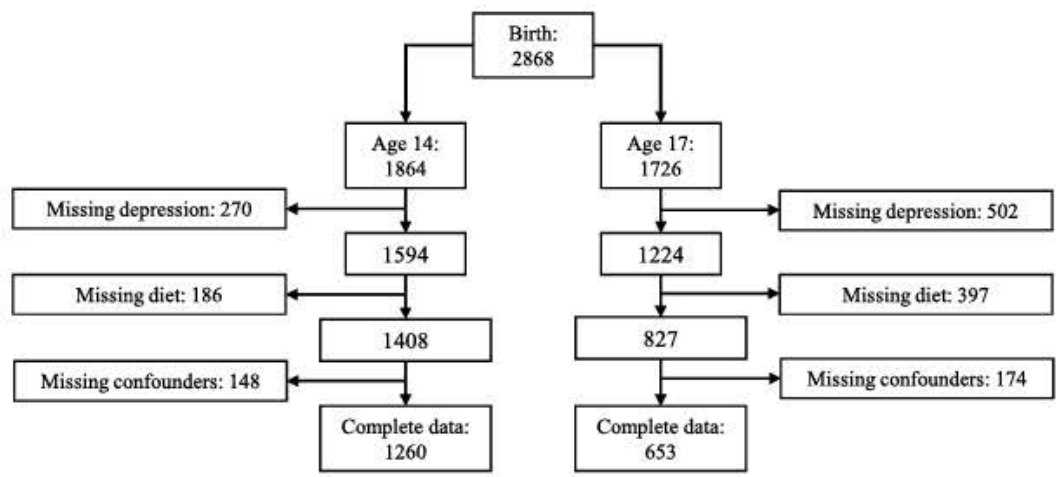

Fig. 1. Number of participants in the Raine Study 14 and 17 year follow ups and number with complete data on depressive symptoms, diet and confounders included in the analysis.

\section{Adiposity measurement}

Participant height (to the nearest $0.1 \mathrm{~cm}$ ) and waist circumfer ence (to the nearest $0.1 \mathrm{~cm}$ ) were measured by trained research ers at each time point and used to calculate waist:height ratio (WHtR, waist circumference $(\mathrm{cm}) /$ height $(\mathrm{cm})$ ). Studies have found that BMI is a poor indicator of body fat percentage ${ }^{(54)}$, including in children and adolescents ${ }^{(55)}$. For this reason, we chose to use WHtR as a marker of adiposity, as it has been shown to be more effective in predicting adiposity than BMI or waist circumference alone in adolescents ${ }^{(56)}$.

\section{Depression measurement}

Depressive symptoms were assessed with the Beck Depression Inventory for Youth (BDI Y) at the 14 and 17 year follow ups. The BDI Y is a self report scale consisting of twenty questions regarding negative feelings in the previous 2 weeks ${ }^{(57)}$. Responses are scored as 03 with the possible answers of: never (0), sometimes (1), often (2) and always (3). Scores are summed, with higher total scores indicating more severe depressive symp toms ${ }^{(58)}$. The minimum valid completion of the BDI Y question naire defined in the handbook is eighteen out of twenty questions $^{(57)}$. Multiple imputation (predictive mean matching) was used for thirty participants who had two or fewer questions unfilled. Raw scores in the range of 060 are converted to T scores standardised by age and sex. The present study uses the T score conversion recommendations for 1518 year olds for consistency ${ }^{(57)}$. Possible scores for girls range from 38 to 97 and scores for boys range from 41 to 100 . Adolescents are cat egorised into groups of severity of depressive symptoms using recommended cut offs ( $<55$ : average, 55 59: mildly elevated, 60 69: moderately elevated and $\geq 70$ : extremely elevated). The groups were combined to create a binary outcome variable of those with average or mildly elevated depressive symptoms $(<60)$ and those with moderately elevated or extremely elevated depressive symptoms $(\geq 60)$, labelled as none/mild ( 0 ) and mod erate/extreme (1). Research using the BDI Y in adolescent girls indicates that this cut off is indicative of clinically meaningful symptoms ${ }^{(59)}$. Additionally, known case analysis of the adult version of the questionnaire (BDI II) in adolescents found that cut off scores approximately equivalent to moderate depressive symptoms had the highest clinical efficiency at identifying those who met diagnostic criteria for major depressive disorder ${ }^{(60,61)}$

\section{Confounders}

Confounders plausibly associated with dietary fibre intake and depression included sex, follow up year (as a marker of age), energy intake, binge drinking, leisure time physical activity, parental education, family income, parental history of mental health problems and family functioning. Energy intake was obtained from the CSIRO FFQ. Dietary misreporting was calcu lated with the Goldberg method ${ }^{(62)}$, using energy intake and BMR as described elsewhere ${ }^{(63)}$.

Lifestyle. At both ages, data on binge drinking and leisure time physical activity were measured from a self report survey. Adolescents were asked if they had ever drunk more than six standard drinks on the same day, with a 'yes' response categor ised as having engaged in binge drinking on one or more occa sions. Leisure time physical activity was defined as the average number of occasions per week adolescents reported spending in vigorous physical activity (causing out of breath and sweating) outside of school hours.

Parental factors. Details of family income were provided by the primary caregiver at the 14 and 17 year follow ups. Parental education was represented by the level of educational attain ment of the highest educated parent as of the 8 year follow up, the most proximal survey of parental education to the 14 and 17 year follow ups. Parents were asked to report in the questionnaires if they had ever been treated for an emotional or mental health problem (other than postnatal depression, yes/ no). Family functioning, a measure of family communication and support, was calculated from the McMaster Family Assessment Device general functioning subscale as completed by the pri mary caregiver ${ }^{(64)}$. Scores were averaged with higher scores on the general functioning subscale representing poorer family functioning. Better family functioning has been associated with greater dietary fibre intake in this population previously ${ }^{(65)}$ and was chosen as a proxy for family environmental factors that may influence both diet and mental health. 


\section{Analysis}

Odds of moderate/extreme depressive symptoms by quartile of dietary fibre intake were determined using mixed effects logistic regression. Although dietary fibre intake differs by sex in this population $^{(65)}$, there was no significant interaction between sex and fibre intake $(P>0.36)$ and therefore sex stratified analy sis was not performed. Significant interactions were found between sex and WHtR, exercise and follow up year. However, addition of these interaction terms to the model had no effect on the OR of depressive symptoms by fibre intake. Additionally, it was not the aim of the paper to examine how the impact of lifestyle factors differs by sex, and therefore they were not included in the final models.

The principle analysis consisted of three models. Model 1 was adjusted for sex, energy intake and dietary misreporting. Model 2 was adjusted for factors in model 1 plus WHtR (per increase of $0 \cdot 1$ ). Model 3 adjusted for factors included in model 2, plus follow up year, binge drinking, physical activity, parental education, fam ily income, parental history of mental health problems and family functioning. All variables except sex and parental education were included as time varying factors across the two follow ups. A sec ondary analysis was restricted to those with data at both the 14 and 17 year follow ups. In this secondary analysis, adolescents who reported moderate/extreme depressive symptoms at the 14 year follow up were excluded to obtain a population with incident depression at age 17 years.

Additional mixed effects logistic regression analyses were conducted to estimate odds of depressive symptoms by quartiles of dietary fibre intake in a subset of the population with hs CRP measurements. hs CRP was added to the final model to test for a mediation effect of inflammation.

The suitability of adjustment for alternative nutrients associ ated with depression was determined by calculating Pearson's correlation coefficients between the candidate nutrients and variance inflation factors (VIF) for the fibre coefficient. VIF can be used to measure multicollinearity or the level of corre lation between variables in regression analysis. Research suggests that VIF as low as two can have an impact on results $^{(66)}$. Nutrients considered were $\mathrm{K}, \mathrm{Mg}, \mathrm{Fe}, \mathrm{Cu}, \mathrm{Zn}$, thi amine, riboflavin, vitamin $\mathrm{B}_{6}$ and folate. As correlation coeffi cients and VIF were high for all nutrients under consideration (online Supplementary Tables S1 and S2), further analysis adjusted for Healthy and Western dietary patterns instead. Although multicollinearity exists between dietary fibre and the Healthy and Western dietary patterns, it is less severe than for individual nutrients and therefore dietary pattern adjustment was the preferred method. VIF for dietary fibre, the Healthy pattern and the Western pattern were $3 \cdot 3,2 \cdot 8$ and 1.4 , respectively. Statistical analysis was performed with $R$ version $3.5 .0^{(67)}$ and $R$ studio version $1.2 .1335^{(68)}$.

\section{Results}

Characteristics of participants overall and with and without mod erate/extreme depressive symptoms at ages 14 and 17 years are shown in Table 1. The proportion of adolescents who were female was higher at the 17 year follow up compared with the
14 year follow up $(54 v, 48 \%)$. A higher percentage of those with moderate/extreme depressive symptoms was female at both ages ( $80 \%$ at age 14 years and $63 \%$ at age 17 years). Those with moderate/extreme depressive symptoms appeared to have poorer lifestyle habits, with lower physical activity and higher rates of binge drinking. They were more likely to have lower family incomes and non formally educated parents with a his tory of mental health problems. Mean dietary fibre intakes were lower in adolescents with moderate/extreme depressive symptoms at both 14 and 17 years (Table 1). The subset of the population with inflammatory data had characteristics similar to those with complete data for all variables (online Supplementary Table S3).

Table 2 shows the proportion of adolescents with moderate/ extreme depressive symptoms at the 14 and 17 year follow ups overall and by quartile of dietary fibre intake. The proportion of adolescents with moderate/extreme depressive symptoms over all was higher at age 17 years $(7 \%)$ than at age 14 years $(3 \%)$. The proportion of adolescents who had moderate/extreme depressive symptoms was lowest in the fourth (highest) quartile of dietary fibre intake at both 14 and 17 years.

Table 3 shows the OR of moderate/extreme depressive symptoms by quartile of overall fibre, cereal fibre, and fruit and vegetable fibre intake relative to the first quartile. In the first model, odds were significantly lower in the fourth quartile of both overall and fruit and vegetable fibre intake and in the third and fourth quartiles of cereal fibre intake compared with the first quartile. After adjusting for WHtR in model 2, there was negli gible change in OR for any fibre type. In model 3 (further adjusted for lifestyle and family factors), significantly lower odds of moderate/extreme depressive symptoms remained in the fourth quartile of overall fibre intake compared with the first quartile (Table 3 ). The expanded results of model 3 in each fibre type are shown in online Supplementary Table S4. In models for overall fibre, cereal fibre, and fruit and vegetable fibre, male was associated with lower odds of moderate/extreme depressive symptoms. For all fibre types, having engaged in binge drinking one or more times and hav ing poorer family functioning were associated with higher odds of moderate/extreme depressive symptoms (online Supplementary Table S4).

OR in paired analysis restricted to those without moderate/ extreme depressive symptoms at the 14 year follow up are shown in Table 4. As in the principle analysis, odds of moderate/ extreme depressive symptoms were significantly lower in the fourth quartile of overall dietary fibre intake compared with the first quartile in all models (Table 4).

Further analysis was performed to adjust for Healthy and Western dietary patterns. Following adjustment for the dietary patterns, the associations with cereal and grain fibre and fruit and vegetable fibre were attenuated in all models, although OR remained consistently lower in the fourth fibre intake quartile (online Supplementary Table S5). For overall fibre, lower OR for moderate/extreme depressive symptoms in the fourth quartile of intake persisted but became non significant in model 3 (Table 5).

Table 6 shows the results of the repeat analysis of odds of moderate/extreme depressive symptoms and overall fibre 
Table 1. Characteristics of included participants from the 14 and 17 year follow ups of the Raine Study overall and by level of depressive symptoms* (Numbers and percentages; mean values and standard deviations)

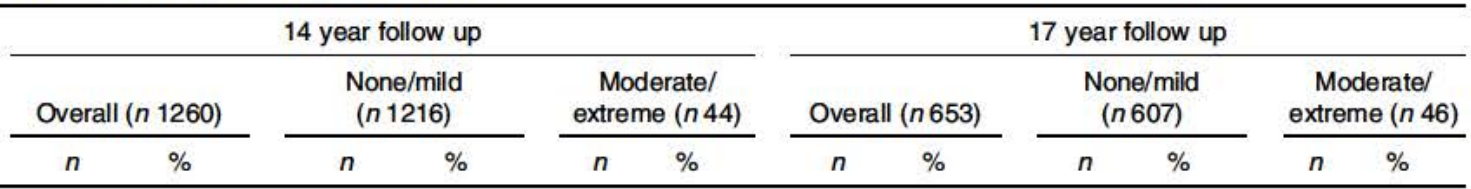

\begin{tabular}{|c|c|c|c|c|c|c|c|c|c|c|c|c|}
\hline \multicolumn{13}{|l|}{ Sex } \\
\hline Female & 611 & 48 & 576 & 47 & 35 & 80 & 354 & 54 & 325 & 54 & 29 & 63 \\
\hline Male & 649 & 52 & 640 & 53 & 9 & 20 & 299 & 46 & 282 & 46 & 17 & 37 \\
\hline \multicolumn{13}{|c|}{ Depressive symptoms (BDI Y) } \\
\hline Average & 1155 & 92 & 1155 & 95 & & & 542 & 83 & 542 & 89 & & \\
\hline Mildly elevated & 61 & 5 & 61 & 5 & & & 65 & 10 & 65 & 11 & & \\
\hline Moderately elevated & 29 & 2 & & & 29 & 66 & 31 & 5 & & & 31 & 67 \\
\hline Extremely elevated & 15 & 1 & & & 15 & 34 & 15 & 2 & & & 15 & 33 \\
\hline \multicolumn{13}{|c|}{ Physical activity (times/week) } \\
\hline None & 108 & 9 & 100 & 8 & 8 & 18 & 131 & 20 & 117 & 19 & 14 & 30 \\
\hline 1 & 255 & 20 & 242 & 20 & 13 & 30 & 136 & 21 & 124 & 20 & 12 & 26 \\
\hline 23 & 475 & 38 & 461 & 38 & 14 & 32 & 220 & 34 & 207 & 34 & 13 & 28 \\
\hline 46 & 270 & 21 & 265 & 22 & 5 & 11 & 108 & 17 & 103 & 17 & 5 & 11 \\
\hline 7 & 152 & 12 & 148 & 12 & 4 & 9 & 58 & 9 & 56 & 9 & 2 & 4 \\
\hline \multicolumn{13}{|l|}{ Binge drinkingt } \\
\hline Never & 1168 & 93 & 1131 & 93 & 37 & 84 & 287 & 44 & 275 & 45 & 12 & 26 \\
\hline Once or more & 92 & 7 & 85 & 7 & 7 & 16 & 366 & 56 & 332 & 55 & 34 & 74 \\
\hline \multicolumn{13}{|l|}{ Parental education } \\
\hline None & 163 & 13 & 153 & 13 & 10 & 23 & 66 & 10 & 60 & 10 & 6 & 13 \\
\hline Year 12 & 126 & 10 & 119 & 10 & 7 & 16 & 55 & 8 & 53 & 9 & 2 & 4 \\
\hline Trade/TAFE & 424 & 24 & 412 & 34 & 12 & 27 & 203 & 31 & 189 & 31 & 14 & 30 \\
\hline University & 547 & 43 & 532 & 44 & 15 & 34 & 329 & 50 & 305 & 50 & 24 & 52 \\
\hline \multicolumn{13}{|l|}{ Family income (AUD) } \\
\hline$\leq 35000$ & 284 & 23 & 270 & 22 & 14 & 32 & 68 & 10 & 61 & 10 & 7 & 15 \\
\hline 3500170000 & 454 & 36 & 443 & 36 & 11 & 25 & 162 & 25 & 150 & 25 & 12 & 26 \\
\hline 70001104000 & 291 & 23 & 278 & 23 & 13 & 30 & 188 & 29 & 175 & 29 & 13 & 28 \\
\hline$>104000$ & 231 & 18 & 225 & 19 & 6 & 14 & 235 & 36 & 221 & 36 & 14 & 30 \\
\hline \multicolumn{13}{|c|}{ Parental history of mental illness } \\
\hline Neither & 850 & 67 & 827 & 68 & 23 & 52 & 422 & 65 & 395 & 65 & 27 & 59 \\
\hline One & 354 & 28 & 336 & 28 & 18 & 41 & 192 & 29 & 178 & 29 & 14 & 30 \\
\hline \multirow[t]{2}{*}{ Both } & 56 & 4 & 53 & 4 & 3 & 7 & 39 & 6 & 34 & 6 & 5 & 11 \\
\hline & Mean & SD & Mean & SD & Mean & SD & Mean & SD & Mean & SD & Mean & SD \\
\hline WHtR & 0.46 & 0.062 & 0.46 & 0.062 & 0.49 & 0.064 & 0.46 & 0.060 & 0.46 & 0.060 & 0.47 & 0.065 \\
\hline Female & 0.46 & 0.061 & 0.46 & 0.060 & 0.49 & 0.065 & 0.46 & 0.061 & 0.46 & 0.064 & 0.49 & 0.069 \\
\hline Male & 0.46 & 0.064 & 0.46 & 0.064 & 0.46 & 0.053 & 0.46 & 0.053 & 0.45 & 0.054 & 0.43 & 0.036 \\
\hline Energy (MJ) & 9.59 & 2.99 & 9.64 & 2.98 & 8.33 & 3.02 & $9 \cdot 26$ & $3 \cdot 26$ & 9.24 & 3.21 & 9.56 & 3.82 \\
\hline Fibre $(\mathrm{g} / \mathrm{d})$ & 23.7 & 8.77 & 23.8 & 8.71 & $21 \cdot 1$ & $10 \cdot 0$ & $22 \cdot 3$ & 8.86 & 22.5 & 8.90 & 19.8 & 7.88 \\
\hline Healthy diet pattem & 0.040 & 0.88 & 0.038 & 0.88 & 0.090 & 0.96 & 0.012 & 0.86 & 0.014 & 0.86 & 0.35 & 0.75 \\
\hline Western diet pattern & 0.0062 & 0.85 & 0.0010 & 0.86 & 0.21 & 0.80 & 0.041 & 0.80 & 0.053 & 0.79 & 0.12 & 0.97 \\
\hline
\end{tabular}

BDI Y, Beck Depression Inventory for Youth; TAFE, technical and further education; WHtR, waist:height ratio.

* Percentages were rounded to the nearest $1 \%$.

t Classified as consuming more than six drinks on one occasion.

Table 2. Proportion of participants with none/mild and moderate/extreme depressive symptoms overall and across quartiles of dietary fibre intake at the 14 and 17 year follow ups of the Raine Study*

(Percentages)

\begin{tabular}{|c|c|c|c|c|}
\hline & \multicolumn{2}{|c|}{14 year follow up ( $n$ 1260) } & \multicolumn{2}{|c|}{17 year follow up ( $n$ 653) } \\
\hline & None/mild (\%) & Moderate/extreme (\%) & None/mild (\%) & Moderate/extreme (\%) \\
\hline Overall & 97 & 3 & 93 & 7 \\
\hline Quartile 1 ( $\leq 17.12 \mathrm{~g} / \mathrm{d})$ & 95 & 5 & 91 & 9 \\
\hline Quartile $2(17.13$ to $\leq 21.92 \mathrm{~g} / \mathrm{d})$ & 95 & 5 & 94 & 6 \\
\hline Quartile $3(21.93$ to $\leq 28.10 \mathrm{~g} / \mathrm{d})$ & 97 & 3 & 90 & 10 \\
\hline Quartile 4 (>28.10 g/d) & 98 & 2 & 97 & 3 \\
\hline
\end{tabular}

* Moderate/extreme depressive symptoms determined by a Beck Depression Inventory for Youth T score of >60.

performed in the subset of participants with hs CRP measure ments. In model 3, odds of moderate/extreme depressive symp toms were significantly lower in the fourth quartile of dietary fibre intake compared with the first. The addition of hs CRP to the final model had little impact on the OR of moderate/extreme depressive symptoms. 
Table 3. Risk of moderate/extreme depressive symptoms per quartile of overall, cereal, and fruit and vegetable fibre intake relative to the first quartile ( $(n 1913)^{*}$ (Odds ratios and $95 \%$ confidence intervals)

\begin{tabular}{|c|c|c|c|c|c|c|c|c|c|}
\hline & \multicolumn{3}{|c|}{ Model 1} & \multicolumn{3}{|c|}{ Model 2} & \multicolumn{3}{|c|}{ Model 3} \\
\hline & OR & $95 \% \mathrm{Cl}$ & $P$ & OR & $95 \% \mathrm{Cl}$ & $P$ & OR & $95 \% \mathrm{Cl}$ & $P$ \\
\hline \multicolumn{10}{|l|}{ Overall fibre } \\
\hline First quartile & \multicolumn{2}{|c|}{ Reference } & & \multicolumn{2}{|c|}{ Reference } & & \multicolumn{2}{|c|}{ Reference } & \\
\hline Second quartile & 0.792 & $0.40,1.59$ & 0.511 & 0.752 & $0.37,1.52$ & 0.425 & 0.894 & $0.44,1.82$ & 0.757 \\
\hline Third quartile & 0.675 & $0.31,1.45$ & 0.315 & 0.669 & $0.31,1.44$ & 0.306 & 0.953 & $0.43,2.10$ & 0.904 \\
\hline Fourth quartile & 0.180 & $0.06,0.54$ & 0.002 & 0.178 & $0.06,0.53$ & 0.002 & 0.273 & $0.09,0.81$ & 0.019 \\
\hline \multicolumn{10}{|l|}{ Cereal fibre } \\
\hline First quartile & \multicolumn{2}{|c|}{ Reference } & & \multicolumn{2}{|c|}{ Reference } & & \multicolumn{2}{|c|}{ Reference } & \\
\hline Second quartile & 0.565 & $0.29,1 \cdot 12$ & 0.101 & 0.559 & $0.28,1.10$ & 0.094 & 0.697 & $0.35,1.39$ & 0.304 \\
\hline Third quartile & 0.393 & $0.18,0.86$ & 0.020 & 0.387 & $0.18,0.85$ & 0.018 & 0.457 & $0.21,1.01$ & 0.053 \\
\hline Fourth quartile & 0.383 & $0.16,0.94$ & 0.036 & 0.397 & $0.16,0.97$ & 0.042 & 0.505 & $0.21,1.23$ & 0.133 \\
\hline \multicolumn{10}{|c|}{ Fruit and vegetable fibre } \\
\hline First quartile & \multicolumn{2}{|c|}{ Reference } & & \multicolumn{2}{|c|}{ Reference } & & \multicolumn{2}{|c|}{ Reference } & \\
\hline Second quartile & 1.07 & $0.56,2.05$ & 0.842 & 1.08 & $0.56,2.07$ & 0.815 & 1.23 & $0.64,2.38$ & 0.536 \\
\hline Third quartile & 0.648 & $0.32,1.32$ & 0.234 & 0.648 & $0.32,1.32$ & 0.233 & 0.854 & $0.41,1.76$ & 0.669 \\
\hline Fourth quartile & 0.328 & $0.14,0.78$ & 0.011 & 0.327 & $0.14,0.78$ & 0.011 & 0.468 & $0.20,1 \cdot 11$ & 0.085 \\
\hline
\end{tabular}

- Comprising 1260 observations from the 14 year follow up and 653 observations from the 17 year follow up. Model 1 adjusted for sex, energy intake and dietary misreporting. Model 2 adjusted for model 1 + waist:height ratio. Model 3 adjusted for model 2 + binge drinking, physical activity, parental education, family income, follow up year, parental history of mental health problems and family functioning.

Table 4. Risk of moderate/extreme depressive symptoms per quartile of overall dietary fibre intake relative to the first quartile in paired analysis restricted to those without moderate/extreme depressive symptoms at age 14 years $(n 1062)^{*}$

(Odds ratios and $95 \%$ confidence intervals)

\begin{tabular}{|c|c|c|c|c|c|c|c|c|c|}
\hline & \multicolumn{3}{|c|}{ Model 1} & \multicolumn{3}{|c|}{ Model 2} & \multicolumn{3}{|c|}{ Model 3} \\
\hline & OR & $95 \% \mathrm{Cl}$ & $P$ & OR & $95 \% \mathrm{Cl}$ & $P$ & OR & $95 \% \mathrm{Cl}$ & $P$ \\
\hline First quartile & \multicolumn{2}{|c|}{ Reference } & \multicolumn{2}{|c|}{ Reference } & \multicolumn{4}{|c|}{ Reference } & \\
\hline Second quartile & 0.320 & $0.10,1.02$ & 0.055 & 0.315 & $0.10,1.01$ & 0.052 & 0.358 & $0.10,1.23$ & 0.103 \\
\hline Third quartile & 0.834 & $0.33,2.12$ & 0.702 & 0.838 & $0.33,2 \cdot 13$ & 0.710 & $1 \cdot 18$ & $0.41,3.39$ & 0.759 \\
\hline Fourth quartile & 0.0645 & $0.01,0.37$ & 0.002 & 0.0647 & $0.01,0.37$ & 0.002 & 0.107 & $0.02,0.69$ & 0.019 \\
\hline
\end{tabular}

* Comprising 531 observations from the 14 year follow up and 531 observations from the 17 yearfollow up. Model 1 adjusted for sex, energy intake and dietary misreporting. Model 2 adjusted for model 1 + waist:height ratio. Model 3 adjusted for model 2 + binge drinking, physical activity, parental education, family income, follow up year, parental history of mental health problems and family functioning.

Table 5. Adjusted risk of moderate/extreme depressive symptoms per quartile of overall fibre intake relative to the first quartile in all models further adjusted for dietary patterns $\left(n 1913^{*}\right)$

(Odds ratios and $95 \%$ confidence intervals)

\begin{tabular}{|c|c|c|c|c|c|c|c|c|c|}
\hline & \multicolumn{3}{|c|}{ Model 1} & \multicolumn{3}{|c|}{ Model 2} & \multicolumn{3}{|c|}{ Model 3} \\
\hline & OR & $95 \% \mathrm{Cl}$ & $P$ & OR & $95 \% \mathrm{Cl}$ & $P$ & OR & $95 \% \mathrm{Cl}$ & $P$ \\
\hline \multicolumn{10}{|l|}{ Dietary fibre intake } \\
\hline First quartile & \multicolumn{2}{|c|}{ Reference } & & \multicolumn{2}{|c|}{ Reference } & & \multicolumn{2}{|c|}{ Reference } & \\
\hline Second quartile & 0.791 & $0.38,1.65$ & 0.533 & 0.752 & $0.36,1.58$ & 0.453 & 0.916 & $0.43,1.94$ & 0.818 \\
\hline Third quartile & 0.681 & $0.27,1.69$ & 0.408 & 0.673 & $0.27,1.68$ & 0.395 & 0.993 & $0.39,2.51$ & 0.987 \\
\hline Fourth quartile & 0.187 & $0.04,0.78$ & 0.022 & 0.183 & $0.04,0.77$ & 0.020 & 0.292 & $0.07,1.20$ & 0.089 \\
\hline Healthy pattern & 1.02 & $0.64,1.64$ & 0.923 & 1.02 & $0.64,1.62$ & 0.945 & 0.954 & $0.59,1.55$ & 0.850 \\
\hline Western pattern & 1.21 & $0.68,2.13$ & 0.515 & 1.15 & $0.65,2.03$ & 0.641 & 0.938 & $0.52,1.69$ & 0.831 \\
\hline
\end{tabular}

* Comprising 1260 observations from the 14 yearfollow up and 653 observations from the 17 year follow up. Model adjusted for sex, energy intake, dietary misreporting, waistheight ratio, binge drinking, physical activity, parental education, family income, follow up year, parental history of mental health problems, family functioning, and healthy and Western dietary pattems.

\section{Discussion}

Our findings suggest that adolescents with the highest intakes of overall dietary fibre have significantly lower odds of clinically relevant moderate/extreme depressive symptoms compared to adolescents with the lowest fibre intakes, before adjustment for overall diet. Although participant numbers were low, simi larly lower odds of depressive symptoms were seen in analysis restricted to adolescents without moderate/extreme depressive symptoms at the 14 year follow up. However, attenuation of the association of dietary fibre with depressive symptoms following adjustment for Healthy and Western dietary patterns suggests that the effect of dietary fibre does not occur independent of other dietary nutrients. Adjusting for hs CRP in a subset of ado lescents with inflammatory data did not alter the results.

Our study is novel in that it is the first to our knowledge to investigate the relationship of dietary fibre and depressive 
Table 6. Risk of moderate/extreme depressive symptoms per quartile of overall dietary fibre intake relative to the first quartile in the subset with data on inflammatory markers $\left(n 1265^{*}\right)$

(Odds ratios and $95 \%$ confidence intervals)

\begin{tabular}{|c|c|c|c|c|c|c|c|c|c|c|c|c|}
\hline & \multicolumn{3}{|c|}{ Model 1} & \multicolumn{3}{|c|}{ Model 2} & \multicolumn{3}{|c|}{ Model 3} & \multicolumn{3}{|c|}{ Model $3+$ hs CRP } \\
\hline & OR & $95 \% \mathrm{Cl}$ & $P$ & OR & $95 \% \mathrm{Cl}$ & $P$ & OR & $95 \% \mathrm{Cl}$ & $P$ & OR & $95 \% \mathrm{Cl}$ & $P$ \\
\hline \multicolumn{13}{|l|}{ First quartile } \\
\hline Second quartile & 0.751 & $0.38,1.49$ & 0.413 & 0.732 & $0.37,1.46$ & 0.374 & 0.856 & $0.42,1.76$ & 0.673 & 0.859 & $0.42,1.77$ & 0.679 \\
\hline Third quartile & 0.740 & $0.35,1.57$ & 0.431 & 0.745 & $0.35,1.58$ & 0.444 & 0.976 & $0.44,2 \cdot 17$ & 0.953 & 0.966 & $0.43,2.15$ & 0.933 \\
\hline Fourth quartile & 0.195 & $0.06,0.59$ & 0.004 & 0.199 & $0.07,0.60$ & 0.004 & 0.264 & $0.08,0.84$ & 0.024 & 0.266 & $0.08,0.85$ & 0.025 \\
\hline
\end{tabular}

hs CRP, high sensitivity $C$ reactive protein.

* Comprising 718 observations from the 14 year follow up and 547 observations from the 17 yearfollow up. Model 1 adjusted for sex, energy intake and dietary misreporting. Model 2 adjusted for model 1 + waistheight ratio. Model 3 adjusted for model $2+$ binge drinking, physical activity, parental education, family income, follow up year, parental history of mental health problems and family functioning.

symptoms in an adolescent population including both females and males. Our finding that the odds of moderate/extreme depressive symptoms were lower in adolescents with the highest dietary fibre intakes before adjustment for dietary factors is con sistent with previous research in adult populations ${ }^{(13-19)}$ and in adolescent girls ${ }^{(20)}$. However, in all such observational studies, there is a possibility of confounding by overall diet. Due to its plant food origins, consumption of dietary fibre occurs with a variety of other nutrients, which may confound the relationship between dietary fibre and depressive symptoms. To our knowl edge, ours is only the second study to attempt to isolate the effect of dietary fibre through adjusting for other dietary factors, with the first by Miki et al. examining depressive symptoms in an adult Japanese population ${ }^{(19)}$. Miki et al. adjusted for intakes of folate, vitamin $\mathrm{B}_{6}$, vitamin $\mathrm{B}_{12}, n 3$ PUFA, Mg and $\mathrm{Zn}$ and found that the association between vegetable and fruit fibre and lower odds of depressive symptoms remained significant after nutrient adjustment $^{(19)}$

In contrast, we chose to look at the confounding effect of pre viously determined dietary patterns instead of individual nutrients. Analysis of dietary patterns has been suggested as a superior method to consider the impacts of overall diet com pared with individual nutrient analysis ${ }^{(69)}$. Dietary pattern adjust ment allowed us to investigate the effects of overall diet while minimising the complications of the high levels of collinearity between dietary fibre and individual nutrients ${ }^{(70)}$. The dietary patterns used in our study were developed with factor analysis based on the CSIRO FFQ at the 14 year follow up. The Healthy pattern includes consumption of high fibre foods such as whole grain cereals, fresh fruit, legumes and vegetables ${ }^{(53)}$. Therefore, adjusting for these patterns may underestimate the effect of fibre. Even with this in mind, substantially lower odds for moderate/ extreme depressive symptoms remain in the fourth quartiles of fibre intake, though not statistically significant, suggesting that dietary fibre intake itself still contributes to lower odds of mod erate/extreme depressive symptoms.

These results of diet adjusted analysis suggest that the asso ciation between a higher dietary fibre intake and lower odds of moderate/extreme depressive symptoms may be more reflective of a high fibre diet with all its accompanying nutrients than of an independent effect of fibre. Other possible pathways from dietary fibre intake to depressive symptoms likely occur in con junction with overall diet.
Given the links between adiposity and depression ${ }^{(29)}$, it is plausible that the association between a diet high in fibre and moderate/extreme depressive symptoms in our study is medi ated by a reduction in adiposity. In univariable analysis, we showed that a higher fibre intake was associated with a smaller WHtR and a smaller WHtR associated with lower OR of moder ate/extreme depressive symptoms. Despite this, adjustment for adiposity using WHtR did not impact on the relationship between dietary fibre intake and depressive symptoms in a mul tivariable model. This suggests that in adolescents, pathways other than adiposity may be involved. Sensitivity analysis adjust ing for BMI instead of WHtR showed little difference in results. It may be that a more precise measure of adiposity such as fat mass or body fat percentage is required to fully capture the impact of adiposity on depressive symptoms.

Dietary fibre may impact depressive symptoms independent of overall diet through the gut microbiota. Dietary fibre remains undigested until it reaches the intestines where it is often fer mented by bacteria. A high fibre intake can encourage the growth of beneficial bacteria $^{(71,72)}$. These bacteria can produce neurotransmitters like gamma amino butyric acid and serotonin, and serotonin's precursor tryptophan ${ }^{(73,74)}$, low concentrations of which have been linked to the pathophysiology of depres $\operatorname{sion}^{(75,76)}$. Additionally, fermentation of dietary fibre by the microbiota creates SCFA ${ }^{(77)}$. SCFA can inhibit histone deacety lases ${ }^{(78)}$, resulting in increased gene expression, including brain derived neurotrophic factor ${ }^{(79)}$. In a previous study of ado lescents, concentrations of brain derived neurotrophic factor, along with other neurotrophins, were significantly lower in clin ically depressed adolescents than in the controls ${ }^{(80)}$. In the same study, lower concentrations of brain derived neurotrophic factor in the depressed adolescents were associated with greater severity of depressive symptoms. Evidence suggests that SCFA can also activate $\mathrm{G}$ protein coupled receptors in the brain ${ }^{(81-83)}$, resulting in norepinephrine release ${ }^{(84,85)}$ which can have anti depressant effects ${ }^{(86)}$.

Another potential mediator of a relationship between a high fibre diet and depressive symptoms is inflammation. Studies have shown that healthy dietary pattems and a high dietary fibre intake associate with lower inflammation ${ }^{(36-38,87)}$, and that high inflamma tion is associated with the presence of depressive symptoms ${ }^{(39-43)}$ Dietary fibre may reduce inflammation through mechanisms such as lowering circulating levels of inflammatory lipopolysaccharide 
by reducing intestinal membrane permeability ${ }^{(88)}$ and $\mathrm{pH}^{(89)}$. High levels of lipopolysaccharide and inflammatory cytokines can alter production and re uptake of neurotransmitters and the function of the hypothalamic pituitary adrenal axis ${ }^{(90,91)}$, which may contrib ute to depression ${ }^{(92)}$. Despite this biological plausibility, our results suggest that reduced hs CRP, as a marker of inflammation, does not mediate the relationship between dietary fibre and depressive symptoms in adolescents.

It is worth noting that the only modifiable lifestyle factor sig nificantly associated with odds of moderate/extreme depressive symptoms in the final model was binge drinking. This is consis tent with previous research linking excessive alcohol consump tion to an increased risk of depression in adolescents and young adults ${ }^{(93)}$. Definitions of excessive, risky or binge drinking vary and have changed over time. Our definition of binge drinking ( $>6$ standard drinks on the same day) differs from the current (2009) Australian National Health and Medical Research Council recommendation of $<4$ standard drinks per $d$ to reduce alcohol related health risks. Therefore, our study may under estimate rates of binge drinking. However, the National Health and Medical Research Council guidelines are designed for adults, with no level of alcohol consumption deemed safe for people under the age of 18 years, and therefore there is no formal definition of adolescent binge drinking. The other fac tors significantly associated with moderate/extreme depressive symptoms were female sex and poorer family functioning.

\section{Strengths and limitations}

In the present study, we used prospective data from two time points in adolescence which is a major strength, as adolescence is an age group where research has so far been lacking. The com prehensive range of data collected from both the adolescents and their parents in the Raine Study allowed adjustment for many potential confounders and testing of mediators. However, the presence of missing data meant that some participants had to be excluded from the study, and the proportion of adolescents with moderate/extreme depressive symptoms was low ( $n 90$, $4.7 \%$ ). To investigate whether the exclusion of some participants biased the results, we performed a sensitivity analysis using inverse probability weighting. The results of the weighted analy sis were consistent with the main analysis, providing some reassurance that exclusion bias did not greatly impact the results. Another strength of the study was the use of two time points, with the same measurements of diet (CSIRO FFQ) and depres sive symptoms (BDI Y) used at each age group. The CSIRO FFQ measures diet from the previous year while the BDI Y deter mines depressive symptoms over the 2 weeks prior to follow up, allowing the examination of the effect of habitual diet on current depressive symptoms. However, due to its retrospective nature the FFQ may be subject to recall bias. As the calculation of the dietary fibre content of diet is dependent on the portion sizes provided by the participant, a reference standard serving size was given for each food item in the questionnaire. To improve accuracy, the primary caregiver assisted in the completion of the FFQ at age 14 years, while it was completed by the adolescent alone at age 17 years. As such, there may have been inconsisten cies in reporting between the two follow ups.
The present study is observational; therefore, we could not confirm any biological causal mechanisms for the association of higher dietary fibre intake with lower odds of moderate/ extreme depressive symptoms. Additionally, we cannot entirely rule out an effect of reverse causality between a high fibre diet and depressive symptoms. However, our results of paired analy sis show that even after excluding participants who had moderate/ extreme depressive symptoms at the 14 year follow up, the highest dietary fibre intakes were associated with lower odds of incident moderate/extreme depressive symptoms at the 17 year follow up. Participant numbers were low ( $n 531,31$ with moderate/extreme depressive symptoms at 17 years), however, and therefore these results cannot be used as strong evidence. We chose not to perform longitudinal analysis using dietary fibre intake at 14 years as the exposure and depressive symptoms at 17 years as the outcome because of a lack of biological plausibility for dietary fibre intake to impact depressive symptoms 3 years later. The BDI Y is a non diagnostic self report questionnaire and as such we cannot deter mine the association between dietary fibre intake and diagnosed clinical depression.

It is difficult if not impossible to isolate the impacts of a single nutrient on depressive symptoms. As dietary fibre is primarily sourced from fruits, vegetables and grains, there is high multicol linearity between fibre and vitamins and minerals that are also present in those foods. As such, we cannot exclude a con founding effect of other nutrients present in a fibre rich diet. Development of a high fibre dietary pattern may be of ben efit for future research regarding the effects of dietary fibre as a component of healthy diet, rather than as an isolated nutrient.

The findings of our study suggest that a good quality, high fibre diet, rather than a high fibre intake alone, may have potential to improve symptoms of depression in adolescents in dependent of adiposity and inflammation. However, our results cannot confirm causality, and further research such as rando mised controlled trials is required to confirm the potential of dietary fibre to impact depressive symptoms. While studies exist on other aspects of diet and mental health in adolescents, to our knowledge, this is the first study to examine the association between dietary fibre intake and depressive symptoms in a sex mixed adolescent cohort. More research in adolescent age groups is important due to the high incidence of mental disorders and associated potential lifelong impacts on health and lifestyle. Further research to determine the utility of dietary fibre in reduc ing depressive symptoms should focus on a high fibre diet rather than simply consuming an allocated quantity of dietary fibre daily. Furthermore, increasing physical activity and discouraging excessive alcohol consumption may be additional effective tar gets for reducing or preventing depressive symptoms in adolescents.

\section{Acknowledgements}

We are grateful to the Raine Study participants and their families, and we thank the Raine Study research staff for cohort coordination and data collection. The core management of the Raine Study is funded by the University of Western Australia, 
Curtin University, Telethon Kids Institute, Women and Infants Research Foundation, Edith Cowan University, Murdoch University, The University of Notre Dame Australia and the Raine Medical Research Foundation. We thank the National Health and Medical Research Council of Australia (NHMRC) for their long term contribution to funding the study over the last 30 years and the Telethon Kids Institute for long term support of the Raine Study.

This work was supported by the Heart Foundation Beyond Blue Strategic Research Program (Oddy et al. ID G08P4036 2009 2012). The Raine Study received funding from the Raine Medical Research Foundation at The University of Western Australia, the NHMRC, the Telstra Research Foundation, the Western Australian Health Promotion Foundation and Australian Rotary Health Research Fund. We acknowledge the Telethon Kids Institute, the Commonwealth Scientific and Industrial Research Organization, the NHMRC Program Grant ID \#003209 and Project Grant \#211912 for supporting the 14 year follow up. Data collection and biological specimens at the 17 year follow up were funded by the NHMRC Program Grant ID 353514 and Project Grant \#403981. T. A. M. is supported by an NHMRC Research Fellowship (ID 1136046) and A. L. is funded by an NHMRC Career Development Fellowship (\#1148793).

Authors' contributions were: O. G. S. formulating the research question, study design, analysis, interpretation, drafting and preparation of final manuscript; M. B. formulating the research question, study design, analysis, interpretation, drafting and review of final manuscript; M. K. and W. H. O. formulating the research question, study design, interpretation, drafting and review of final manuscript; T. A. O., T. A. M., L. J. B. and A. L. study design, interpretation and review of final manuscript.

All authors declare that there are no potential or existing conflicts of interest.

\section{Supplementary material}

For supplementary material referred to in this article, please visit https://doi.org/10.1017/S0007114520003426

\section{References}

1. James SL, Abate D, Abate KH, et al. (2018) Global, regional, and national incidence, prevalence, and years lived with disability for 354 diseases and injuries for 195 countries and territories, 1990 2017: a systematic analysis for the Global Burden of Disease Study 2017. Lancet 392, 17891858.

2. Gore FM, Bloem PJ, Patton GC, et al. (2011) Global burden of disease in young people aged 1024 years: a systematic analy sis. Lancet 377, 20932102.

3. Khalid S, Williams CM \& Reynolds SA (2016) Is there an asso ciation between diet and depression in children and adoles cents? A systematic review. BrJ Nutr 116, 20972108.

4. Khosravi M, Sotoudeh G, Majdzadeh R, et al. (2015) Healthy and unhealthy dietary patterns are related to depression: a case control study. Psychiatry Investig 12, 434442.

5. LaiJS, Hiles S, Bisquera A, et al. (2014) A systematic review and meta analysis of dietary patterns and depression in community dwelling adults. Am J Clin Nutr 99, 181197.
6. Saghafian F, Malmir H, Saneei P, et al. (2018) Fruit and vegeta ble consumption and risk of depression: accumulative evi dence from an updated systematic review and meta analysis of epidemiological studies. BrJ Nutr 119, 10871101.

7. Li Y, Lv MR, Wei YJ, et al. (2017) Dietary patterns and depres sion risk: a meta analysis. Psychiatry Res 253, 373382.

8. Molendijk M, Molero P, Ortuño Sánchez Pedreño F, et al. (2018) Diet quality and depression risk: a systematic review and dose response meta analysis of prospective studies. J Affect Disord 226, 346354.

9. Lassale C, Batty GD, Baghdadli A, et al. (2019) Healthy dietary indices and risk of depressive outcomes: a systematic review and meta analysis of observational studies. Mol Psychiatry 24, 965986 .

10. Firth J, Marx W, Dash S, et al. (2019) The effects of dietary improvement on symptoms of depression and anxiety: a meta analysis of randomized controlled trials. Psychosom Med 81, 265280.

11. Opie RS, O'Neil A, Itsiopoulos C, et al. (2015) The impact of whole of diet interventions on depression and anxiety: a sys tematic review of randomised controlled trials. Public Health Nutr 18, 20742093.

12. Agarwal U, Mishra S, Xu J, et al. (2015) A multicenter random ized controlled trial of a nutrition intervention program in a multiethnic adult population in the corporate setting reduces depression and anxiety and improves quality of life: the GEICO study. Am J Health Promot 29, 245254.

13. Gopinath B, Flood VM, Burlutksy G, et al. (2016) Association between carbohydrate nutrition and prevalence of depressive symptoms in older adults. Br J Nutr 116, 21092114.

14. Gangwisch JE, Hale L, Garcia L, et al. (2015) High glycemic index diet as a risk factor for depression: analyses from the Women's Health Initiative. Am J Clin Nutr 102, 454463.

15. Oishi J, Doi H \& Kawakami N (2009) Nutrition and depressive symptoms in community dwelling elderly persons in Japan. Acta Med Okayama 63,9 17.

16. Woo J, Lynn H, Lau WY, et al. (2006) Nutrient intake and psychological health in an elderly Chinese population. Int J Geriatr Psychiatry 21, 10361043.

17. Fang CY, Egleston BL, Gabriel KP, et al. (2013) Depressive symptoms and serum lipid levels in young adult women. JBehav Med 36, 143152.

18. Xu H, Li S, Song X, et al. (2018) Exploration of the association between dietary fiber intake and depressive symptoms in adults. Nutrition 54, 4853 .

19. Miki T, Eguchi M, Kurotani K, et al. (2016) Dietary fiber intake and depressive symptoms in Japanese employees: the Furukawa Nutrition and Health Study. Nutrition 32, 584589.

20. Kim TH, Choi JY, Lee HH, et al. (2015) Associations between dietary pattern and depression in Korean adolescent girls. J Pediatr Adolesc Gynecol 28, 533537.

21. Belfer ML (2008) Child and adolescent mental disorders: the magnitude of the problem across the globe. J Child Psychol Psychiatry 49, 226236.

22. Kim Cohen J, Caspi A, Moffitt TE, et al. (2003) Prior juvenile diagnoses in adults with mental disorder: developmental follow back of a prospective longitudinal cohort. Arch Gen Psychiatry 60, 709717 .

23. Thapar A, Collishaw S, Pine DS, et al. (2012) Depression in ado lescence. Lancet 379, 10561067.

24. Fergusson DM, Horwood LJ, Ridder EM, et al. (2005) Subthreshold depression in adolescence and mental health out comes in adulthood. Arch Gen Psychiatry 62, 6672.

25. Fröjd SA, Nissinen ES, Pelkonen MUI, et al. (2008) Depression and school performance in middle adolescent boys and girls. J Adolesc 31, 485498 . 
26. Glied S \& Pine DS (2002) Consequences and correlates of ado lescent depression. JAMA Pediatr 156, 10091014.

27. Hetrick SE, Parker AG, Hickie IB, et al. (2008) Early identifica tion and intervention in depressive disorders: towards a clinical staging model. Psychother Psychosom 77, 263270.

28. Hetrick SE, Cox GR, Witt KG et al. (2016) Cognitive behavioural therapy (CBT), third wave CBT and interpersonal therapy (IPT) based interventions for preventing depression in children and adolescents. Cochrane Database Syst Rev, issue 8, CD003380.

29. Zhao G, Ford ES, Li C, et al. (2011) Waist circumference, abdominal obesity, and depression among overweight and obese U.S. adults: National Health and Nutrition Examination Survey 2005 2006. BMC Psychiatry 11, 130.

30. Sanderson K, Patton GC, McKercher C, et al. (2011) Overweight and obesity in childhood and risk of mental disorder: a 20 year cohort study. Aust N Z J Psychiatry 45, 384392

31. Parker G \& Crawford J (2007) Chocolate craving when depressed: a personality marker. BrJPsychiatry 191, 351352

32. Whitaker KM, Sharpe PA, Wilcox S, et al. (2014) Depressive symptoms are associated with dietary intake but not physical activity among overweight and obese women from disadvan taged neighborhoods. Nutr Res 34, 294301.

33. Miller GE, Freedland KE, Carney RM, et al. (2003) Pathways linking depression, adiposity, and inflammatory markers in healthy young adults. Brain Behav Immun 17, 276285.

34. Shelton RC \& Miller AH (2010) Eating ourselves to death (and despair): the contribution of adiposity and inflammation to depression. Prog Neurobiol 91, 275299.

35. Visser M, Bouter LM, McQuillan GM, et al. (1999) Elevated $\mathrm{C}$ reactive protein levels in overweight and obese adults. JAMA 282, 21312135.

36. Wannamethee SG, Whincup PH, Thomas MC, et al. (2009) Associations between dietary fiber and inflammation, hepatic function, and risk of type 2 diabetes in older men: potential mechanisms for the benefits of fiber on diabetes risk. Diabetes Care 32, 18231825

37. Ajani UA, Ford ES \& Mokdad AH (2004) Dietary fiber and $\mathrm{C}$ reactive protein: findings from National Health and Nutrition Examination Survey data. J Nutr 134, 11811185.

38. Parikh S, Pollock NK, Bhagatwala J, et al. (2012) Adolescent fiber consumption is associated with visceral fat and inflamma tory markers. J Clin Endocrinol Metab 97, E1451 1457.

39. Martinac M, Babic D, Bevanda M, et al. (2017) Activity of the hypothalamic pituitary adrenal axis and inflammatory media tors in major depressive disorder with or without metabolic syndrome. Psychiatr Danub 29, 3950.

40. Verduijn J, Milaneschi Y, Schoevers RA, et al. (2015) Pathophysiology of major depressive disorder: mechanisms involved in etiology are not associated with clinical progres sion. Transl Psychiatry 5, e649.

41. Panagiotakos DB, Pitsavos C, Chrysohoou C, et al. (2004) Inflammation, coagulation, and depressive symptomatology in cardiovascular disease free people; the ATTICA study. Eur Heart J 25, 492499.

42. Howren MB, Lamkin DM \& Suls J (2009) Associations of depres sion with $C$ reactive protein, IL 1 , and $I L$ 6: a meta analysis. Psychosom Med 71, 171186.

43. Dowlati Y, Hermann N, Swardfager W, et al. (2010) A meta analysis of cytokines in major depression. Biol Psychiatry 67, 446457.

44. Jacka FN, Cherbuin N, Anstey KJ, et al. (2015) Does reverse cau sality explain the relationship between diet and depression? $J$ Affect Disord 175, 248250.

45. Straker L, Mountain J, Jacques A, et al. (2017) Cohort profile: the Western Australian Pregnancy Cohort (Raine) Study Generation 2. Int J Epidemiol 46, 1384 1385j.
46. White SW, Eastwood PR, Straker LM, et al. (2017) The Raine study had no evidence of significant perinatal selection bias after two decades of follow up: a longitudinal pregnancy cohort study. BMC Pregnancy Cbildbirth 17, 207.

47. Ambrosini GL, de Klerk NH, O'Sullivan TA, et al. (2009) The reliability of a food frequency questionnaire for use among ado lescents. Eur J Clin Nutr 63,1251 1259.

48. Ambrosini GL, O'Sullivan TA, de Klerk NH, et al. (2011) Relative validity of adolescent dietary patterns: a comparison of a FFQ and 3 d food record. BrJ Nutr 105, 625633.

49. Lassale C, Guilbert C, Keogh J, et al. (2009) Estimating food intakes in Australia: validation of the Commonwealth Scientific and Industrial Research Organisation (CSIRO) food frequency questionnaire against weighed dietary intakes. $J$ Hum Nutr Diet 22, 559566.

50. Zhang G, Ding H, Chen $\mathrm{H}$, et al. (2012) Thiamine nutritional status and depressive symptoms are inversely associated among older Chinese adults. J Nutr 143, 5358.

51. Murakami K, Miyake Y, Sasaki S, et al. (2010) Dietary folate, riboflavin, vitamin B 6, and vitamin B 12 and depressive symp toms in early adolescence: the Ryukyus Child Health Study. Psychosom Med 72, 763768.

52. Jacka FN, Maes M, Pasco JA, et al. (2012) Nutrient intakes and the common mental disorders in women. J Affect Disord 141, 7985 .

53. Ambrosini GL, Oddy WH, Robinson M, et al. (2009) Adolescent dietary patterns are associated with lifestyle and family psycho social factors. Public Health Nutr 12, 18071815.

54. Nuttall FQ (2015) Body mass index: obesity, BMI, and health: a critical review. Nutr Today 50, 117128.

55. Widhalm K \& Schönegger K (1999) BMI: does it really reflect body fat mass? J Pediatr 134, 522.

56. Brambilla P, Bedogni G, Heo M, et al. (2013) Waist circumfer ence to height ratio predicts adiposity better than body mass index in children and adolescents. Int $J$ Obes 37, 943946.

57. Beck JS, Beck AT \& Jolly JB (2005) Beck Youth Inventories for Children and Adolescents Manual. San Antonio, TX: Pearson.

58. Steer RA, Kumar G, Beck AT, et al. (2005) Dimensionality of the Beck Youth Inventories with child psychiatric outpatients. J Psychopathol Behav Assess 27, 123131.

59. Stapleton LM, Sander JB \& Stark KD (2007) Psychometric prop erties of the Beck Depression Inventory for Youth in a sample of girls. Psychol Assess 19, 230235.

60. Krefetz DG, Steer RA, Gulab NA, et al. (2002) Convergent val idity of the Beck Depression Inventory II with the Reynolds Adolescent Depression Scale in psychiatric inpatients. $J$ Pers Assess 78, 451460.

61. Kumar G, Steer RA, Teitelman KB, et al. (2002) Effectiveness of Beck Depression Inventory II subscales in screening for major depressive disorders in adolescent psychiatric inpatients. Assessment 9, 164170.

62. Goldberg GR, Black AE, Jebb SA, et al. (1991) Critical evalu ation of energy intake data using fundamental principles of energy physiology: 1 . Derivation of cut off limits to identify under recording. Eur J Clin Nutr 45, 569581.

63. Appannah G, Pot GK, O'Sullivan TA, et al. (2014) The reliability of an adolescent dietary pattern identified using reduced rank regression: comparison of a FFQ and $3 \mathrm{~d}$ food record. BrJ Nutr 112, 609615 .

64. Epstein NB, Baldwin LM \& Bishop DS (1983) The McMaster family assessment device. J Marital Fam Ther 9, 171180.

65. Swann OG, Breslin M, Kilpatrick M, et al. (2020) Differences in dietary fibre intake and associated familial factors in a longi tudinal study at two time points across adolescence. Public Health Nutr 23, 25392547. 
66. Graham $\mathrm{MH}$ (2003) Confronting multicollinearity in ecological multiple regression. Ecology 84, 28092815.

67. R Core Team (2018) $R$ : A Language and Environment for Statistical Computing, 3.5.0 ed. Vienna: R Foundation for Statistical Computing

68. RStudio (2018) RStudio, 1.1.453 ed. Boston, MA: RStudio.

69. Hu FB (2002) Dietary pattern analysis: a new direction in nutri tional epidemiology. Curr Opin Lipidol 13, 39.

70. McGee D, Reed D \& Yano K (1984) The results of logistic analy ses when the variables are highly correlated: an empirical example using diet and CHD incidence. J Chronic Dis 37, 713719.

71. Xu Z \& Knight R (2015) Dietary effects on human gut micro biome diversity. Br J Nutr 113, Suppl. 1, S1 S5.

72. Tap J, Furet JP, Bensaada M, et al. (2015) Gut microbiota rich ness promotes its stability upon increased dietary fibre intake in healthy adults. Environ Microbiol 17, 49544964.

73. Lyte M (2011) Probiotics function mechanistically as delivery vehicles for neuroactive compounds: microbial endocrinology in the design and use of probiotics. Bioessays 33, 574581.

74. O'Mahony SM, Clarke G, Borre YE, et al. (2015) Serotonin, tryp tophan metabolism and the brain gut microbiome axis. Behav Brain Res 277, 3248.

75. Bell C, Abrams J \& Nutt D (2001) Tryptophan depletion and its implications for psychiatry. BrJ Psychiatry 178, 399405.

76. Luscher B \& Fuchs T (2015) GABAergic control of depression related brain states. Adv Pharmacol 73, 97144.

77. Cummings JH (1981) Short chain fatty acids in the human colon. Gut 22, 763779 .

78. Sealy L \& Chalkley R (1978) The effect of sodium butyrate on histone modification. Cell 14, 115121.

79. Kim HJ, Leeds P \& Chuang D M (2009) The HDAC inhibitor, sodium butyrate, stimulates neurogenesis in the ischemic brain J Neurochem 110, 12261240.

80. Pallavi P, Sagar R, Mehta M, et al. (2013) Serum neurotrophic factors in adolescent depression: gender difference and corre lation with clinical severity. J Affect Disord 150, 415423.

81. Tarini J \& Wolever TM (2010) The fermentable fibre inulin increases postprandial serum short chain fatty acids and reduces free fatty acids and ghrelin in healthy subjects. Appl Pbysiol Nutr Metab 35, 916.
82. Pomare EW, Branch WJ \& Cummings JH (1985) Carbohydrate fermentation in the human colon and its relation to acetate concentrations in venous blood. J Clin Invest $\mathbf{7 5}$, 14481454

83. Scheppach W, Pomare EW, Elia M, et al. (1991) The contribution of the large intestine to blood acetate in man. Clin Sci $\mathbf{8 0}$, 177182.

84. Kimura I, Inoue D, Maeda T, et al. (2011) Short chain fatty acids and ketones directly regulate sympathetic nervous system via $\mathrm{G}$ protein coupled receptor 41 (GPR41). Proc Natl Acad Sci U S A 108, 80308035 .

85. Inoue D, Kimura I, Wakabayashi M, et al. (2012) Short chain fatty acid receptor GPR41 mediated activation of sympathetic neurons involves synapsin $2 \mathrm{~b}$ phosphorylation. FEBS Lett 586, 15471554 .

86. Moret C\& Briley M(2011) The importance of norepinephrine in depression. Neuropsychiatr Dis Treat 7, Suppl. 1, S9 S13.

87. Silveira BKS, Oliveira TMS, Andrade PA, et al. (2018) Dietary pattern and macronutrients profile on the variation of inflam matory biomarkers: scientific update. Cardiol Res Pract 2018, 4762575.

88. Peng L, Li Z R, Green RS, et al. (2009) Butyrate enhances the intestinal barrier by facilitating tight junction assembly via acti vation of AMP activated protein kinase in Caco 2 cell mono layers. J Nutr 139, 16191625.

89. den Besten G, van Eunen K, Groen AK, et al. (2013) The role of short chain fatty acids in the interplay between diet, gut micro biota, and host energy metabolism. J Lipid Res 54, 23252340.

90. Leonard B \& Maes M (2012) Mechanistic explanations how cell mediated immune activation, inflammation and oxidative and nitrosative stress pathways and their sequels and concomitants play a role in the pathophysiology of unipolar depression. Neurosci Biobehav Rev 36, 764785.

91. Krishnan V \& Nestler EJ (2008) The molecular neurobiology of depression. Nature 455, 894902.

92. Werner F M \& Coveñas R (2010) Classical neurotransmitters and neuropeptides involved in major depression: a review. Int J Neurosc 120, 455470.

93. Fergusson DM, Boden JM \& Horwood L (2009) Tests of causal links between alcohol abuse or dependence and major depres sion. Arch Gen Psychiatry 66, 260266. 